\title{
Prediction of heat-induced collagen shrinkage by use of second harmonic generation microscopy
}

\author{
Sung-Jan Lin \\ National Taiwan University Hospital \\ and College of Medicine \\ Department of Dermatology \\ Taipei, Taiwan \\ and \\ National Taiwan University Hospital \\ Department of Dermatology \\ Yulin Branch \\ Douliu, Yulin, Taiwan
}

\section{Wen Lo}

National Taiwan University

Department of Physics

Taipei, Taiwan

\author{
Hsin-Yuan Tan \\ Chang Gung Memorial Hospital \\ Department of Ophthalmology \\ Linko, Taiwan \\ and \\ National Taiwan University \\ Institute of Biomedical Engineering \\ Taipei, Taiwan
}

\section{Jung-Yi Chan}

National Taiwan University Hospital

College of Medicine

Department of Dermatology

Taipei, Taiwan and

Cathay General Hospital

Department of Dermatology

Taipei, Taiwan

\section{Wei-Liang Chen}

National Taiwan University

Department of Physics

Taipei, Taiwan

\section{Shiou-Han Wang}

National Taiwan University Hospital

College of Medicine

Department of Dermatology

Taipei, Taiwan

\section{Yen Sun}

National Taiwan University

Department of Physics

Taipei, Taiwan

\section{Wei-Chou Lin}

National Taiwan University Hospital

Department of Pathology

Taipei, Taiwan

Address all correspondence to Shiou-Hwa Jee, Department of Dermatology, National Taiwan University Hospital, Taipei 100, Taiwan; Tel: 886-2-23562141; Fax: 886-2-2393-4177; and Chen-Yuan Dong, Department of Physics, National Taiwan University, Taipei 106, Taiwan; Tel: 886-2-3366-5155; Fax: 886-2-2363-9984

\author{
Jau-Shiuh Chen \\ Chih-Jung Hsu \\ Jeng-Wei Tjiu \\ Hsin-Su Yu \\ Shiou-Hwa Jee \\ National Taiwan University Hospital \\ and College of Medicine \\ Department of Dermatology \\ Taipei, Taiwan
}

\author{
Chen-Yuan Dong \\ National Taiwan University \\ Department of Physics \\ Taipei, Taiwan
}

\begin{abstract}
Collagen shrinkage associated with denaturation from thermal treatment has a number of important clinical applications. However, individualized treatment is hindered by the lack of reliable noninvasive methods to monitor the process of collagen denaturation. We investigate the serial changes of collagen denaturation from thermal treatment of rat tail tendons at $58{ }^{\circ} \mathrm{C}$ by use of second harmonic generation ( $\mathrm{SHG}$ ) microscopy. We find that rat tail tendon shrinks progressively from 0 to 9 min of thermal treatment, and remains unchanged in length upon further thermal treatment. The SHG intensity also decreases from 0 to $9 \mathrm{~min}$ of thermal treatment and becomes barely detectable from further thermal treatment. Collagen shrinkage and the SHG intensity are well correlated in a linear model. In addition, SHG imaging reveals a tiger-taillike pattern of collagen denaturation. The bands of denatured collagen progressively widen from increased thermal treatment and completely replace the adjacent bands of normal collagen after 9 min of thermal treatment. Our results show that collagen denaturation in rat tail tendon from thermal treatment is inhomogeneous, and that SHG intensity can be used to predict the degree of thermally induced collagen shrinkage. With additional development, this approach has the potential to be used in biomedical applications. ๑ 2006 Society of Photo-Optical Instrumentation Engineers. [DOI: 10.1117/1.2209959]
\end{abstract}

Keywords: second harmonic generation; collagen; denaturation; shrinkage; thermal.

Paper 05388R received Dec. 23, 2005; revised manuscript received Mar. 28, 2006; accepted for publication Mar. 28, 2006; published online Jun. 20, 2006.

\section{Introduction}

Various clinical procedures aimed at shrinking collagen have been achieved by thermal treatments. ${ }^{1-3}$ For example, in the field of dermatology, advances in heating modalities, such as the use of various types of lasers, intense pulse light, and radiofrequency, have led to their applications in antiaging

1083-3668/2006/11(3)/034020/6/\$22.00 @ 2006 SPIE 
therapies for tightening slack skin. ${ }^{3-11}$ Despite the wide application of heat-induced collagen shrinkage, serial collagen denaturation during the shrinkage process and the subsequent tissue remodeling have not been investigated in detail due to the lack of an appropriate monitoring tool. Without real-time monitoring of the collagen denaturation process from thermal treatment, identification of appropriate adjustable clinical parameters will continue to be based on trial-and-error practice and years of accumulative experience. ${ }^{12}$ At the present, controlled collagen shrinkage by thermal treatment can only be prematurely judged on the basis of subjective clinical assessment by physicians. Hence, there is a need for a monitoring tool to quantify the effects of heating parameters on treatment results. Since tissue condition of each patient varies, real-time monitoring of treatment can also lead to effective individualized treatment.

Collagen 1 is the most abundant protein in various tissues. Structurally, it is a triple helix, composed of three polypeptide chains, each of approximately $300 \mathrm{~nm}$ in length. ${ }^{12,13}$ After being secreted into the extracellular space, the molecules are aligned into highly organized structures, such as microfibrils, fibrils, and fascicles. It has been shown that collagen denatures under thermal treatment and the denaturation is accompanied by a gross shrinkage. ${ }^{14-16}$ Although the detailed coupled mechanisms underlying collagen denaturation and gross fiber shrinkage are not fully understood, it is believed that heating breaks the hydrogen bonds that stabilize the three polypeptide chains in the native triple helical molecular structure, resulting in an irreversible transformation of collagen molecules into a random coil, and the disruption of the collagen molecular structure leads to the gross shrinkage of collagenous tissue. ${ }^{12,17}$

With the advent of imaging technology such as reflectance confocal microscopy and optical coherence tomography, noninvasive tissue imaging with enhanced resolution in the clinical setting has become possible. ${ }^{18-20}$ However, optimal imaging of the structural changes of collagen molecules cannot be achieved by these techniques. Recently, the nonlinear optical effect of second harmonic generation (SHG) has been extensively used in biomedical imaging. ${ }^{21-28}$ Biological structures lacking a central symmetry are effective in generating SHG signals. Specifically, the interaction of incident lasers with noncentrosymmetric biological structures leads to the generation of photons at a wavelength of exactly half the wavelength of the incident light. ${ }^{21,23}$ Unlike fluorescence excitation, no molecular transition is involved in the generation of the second harmonic signal. Therefore, sample photodamage is significantly reduced. A number of biological materials such as collagen, microtubules, and muscle fibers are effective in generating SHG, and since SHG is structurally sensitive, transitions of biomolecular structures can be monitored. ${ }^{21-24,27,29} \mathrm{It}$ has been shown that the modulation of collagen by cancer cells can be noninvasively monitored in animal models using this technique. ${ }^{22}$ We have also shown that SHG can be used to monitor the structural transition of collagen at various temperatures and to quantify dermal changes associated with photoaging. 30,31

In this work, we employed SHG microscopy in the investigation of collagen shrinkage and denaturation under an isothermal condition. We obtained the serial SHG images and intensities from rat tail tendon under thermal treatment of
$58{ }^{\circ} \mathrm{C}$ for 0 to $15 \mathrm{~min}$. The shrinkage of rat tail tendon from the same thermal treatment is also measured.

\section{Materials and Methods}

\subsection{Second Harmonic Generation Microscope}

The second harmonic imaging system used in this study is a modified version of a home-built laser scanning microscopic imaging system based on an upright microscope (E800, Nikon, Japan) described previously. ${ }^{30}$ A titanium-sapphire (Ti: sapphire Tsunami, Spectra Physics) laser is used as the excitation source. The 760-nm output of the Ti:sapphire laser is scanned in the focal plane by a galvanometer-driver $x-y$ mirror scanning system (Model 6220, Cambridge Technology, Cambridge, Massachusetts). Prior to entering the upright microscope, the laser is beam expanded to ensure overfilling of the objective's back aperture. For high resolution imaging, a high numerical aperture, oil immersion objective (S Fluor $40 \times$, NA 1.3, Nikon) is selected for SHG microscopy. To direct the expanded laser spot to the sample, a short-pass dichroic mirror (700DCSPXRUV-3p, Chroma Technology, Brattleboro, Vermont) is used to reflect the incident excitation laser source. To ensure even excitation of collagen fibers at different orientations, a $\lambda / 4$ wave plate is used to convert the linearly polarized Ti: sapphire laser beam into one with circular polarization. The average laser power at the sample is $3.1 \mathrm{~mW}$, and the SHG signal generated at this power is found to be within the quadratic dependence region of the SHG signal to the excitation power. The generated SHG signal is then collected in the backscattering geometry, where the dichroic mirror, a short-pass filter (E680SP, Chroma Technology), and a 380-nm bandpass filter (HQ380/20, Chroma Technology) are used to isolate the SHG signal. The signal photons are processed by a single-photon counting PMT (R7400P, Hamamatsu, Japan) and a home-built discriminator.

\subsection{Preparation of Rat Tail Tendon}

Rat tail tendon is chosen in this study because the organization of rat tail tendon is relatively simple, consisting of bundles of parallel collagen fascicles oriented along the long axis of the tendon. ${ }^{26,31}$ The experiment involving tissue from animals has been approved by our Institutional Review Board. In our study, tail tendons of a Winstar rat are acquired from the same rat tail in the same experiment. The tendons are cut into strips $3 \mathrm{~cm}$ in length and placed in phosphate buffered saline (PBS) prior to being subjected to $58{ }^{\circ} \mathrm{C}$ thermal baths. The temperature is selected because collagen denaturation at this temperature is observed in our previous work. In this manner, we can ensure rapid and uniform heating and cooling of the tendon specimens when placing them into and removing them from the thermal bath. The periods of thermal treatment range from 0 to 15 min with a 90 -s increment. At the end of thermal treatment, the tendon specimens are removed and the length of each specimen is measured. Then, specimens at each point in time are mounted on a glass slide and covered with a number 1.5 cover glass for viewing. We acquired second harmonic signals and images of the tendons with different periods of thermal treatment. To gain a thorough understanding of the effects of heating on collagen, five randomly chosen large area scans composed of $6 \times 6$ arrays of 


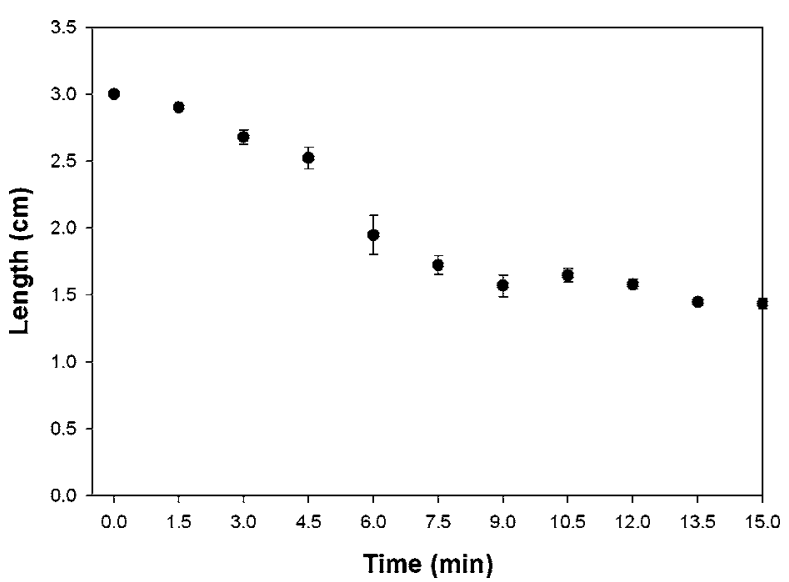

Fig. 1 Changes of lengths of rat tail tendon from thermal treatment at $58{ }^{\circ} \mathrm{C}$. Error bars represent calculated standard deviations.

neighboring SHG images are acquired and assembled in each specimen. The average SHG signal per pixel is computed and plotted. To eliminate the effects of sample scattering or refractive-index-induced spherical aberration on the measured SHG signals, we only acquire the SHG images at the surface of the tendon specimen. After the imaging process is completed, the specimen is fixed in buffered formalin solution (10\% in PBS) and further processed for histological examination (hematoxylin and eosin stains).

\section{Results}

\subsection{Shrinkage of Collagen by Thermal Treatment}

Figure 1 shows the serial changes of the length of rat tail tendon along the longitudinal axis after thermal treatment for $0,1.5,3,4.5,6,7.5,9,10.5,12,13.5$, and 15 min. Examinations of Fig. 1 show that rat tail tendon progressively shortens from 0 to 9 min of thermal treatment. Beyond 9 min of thermal treatment, the length of rat tail tendon remains almost constant from further thermal treatment. This suggests that there is a limit to which thermal treatment can be used to shrink collagen fibers.

\subsection{Changes of Second Harmonic Generation Intensity from Thermal Treatment}

The corresponding changes of SHG intensity during thermal treatment are shown in Fig. 2. Interestingly, SHG intensities also show a decreasing trend from 0 to 9 min of thermal treatment (Fig. 2). The SHG intensities are almost undetectable at $9 \mathrm{~min}$ and remain at andetectable baseline value during further thermal treatment. Hence, the collagen structure in rat tail tendons responsible for $\mathrm{SHG}$ is completely disrupted after thermal treatment at $58{ }^{\circ} \mathrm{C}$ for $9 \mathrm{~min}$.

By comparing Figs. 1 and 2, several interesting results can be obtained. Both the length of collagen fibers and overall SHG intensities decrease with the duration of thermal treatment for up to $9 \mathrm{~min}$. After heating the samples for $9 \mathrm{~min}$ and beyond, SHG diminishes to nearly undetectable levels. At the same time, collagen fibers also shrink to a terminal length. Thermal treatment beyond 9 min fails to shorten collagen fibers. This result suggests that collagen shrinkage by thermal

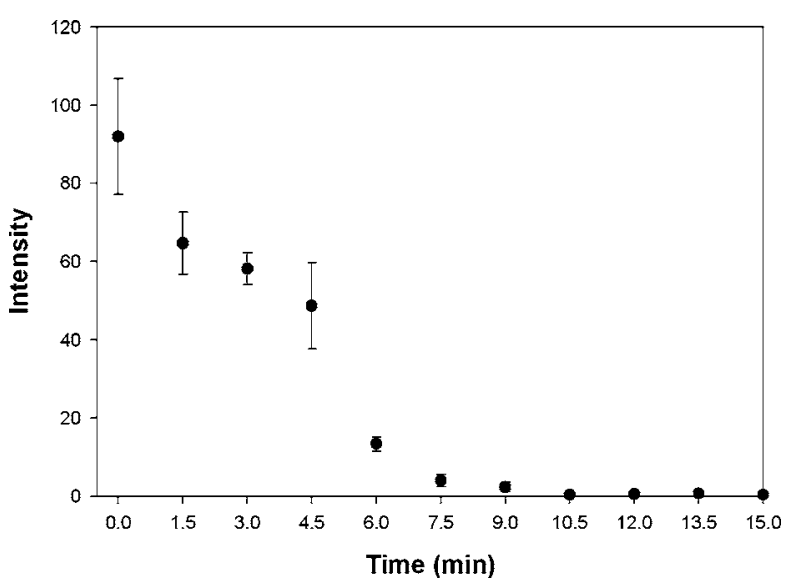

Fig. 2 Changes of SHG intensities of rat tail tendon from thermal treatment at $58{ }^{\circ} \mathrm{C}$. Error bars represent calculated standard deviations.

treatment cannot be efficiently achieved after the collagen structure responsible for SHG is completely disrupted.

\subsection{Mechanisms of Collagen Thermal Denaturation Revealed by Second Harmonic Generation Imaging}

The SHG images of specimens after thermal treatment of various periods are shown in Fig. 3. In contrast to the homogeneous decrease in SHG intensity across the entire specimen, the SHG images show a progressive degradation of collagen [Figs. 3(b)-3(e)]. This result is compatible with the result found in histological examination [Figs. 4(b)-4(e)] in which alternated bands of intact fibrous collagen structures and homogenized collagen are revealed. In comparison with the untreated specimen shown in Fig. 3(a), collagen fibers become wavy and narrow bands of denatured collagen can be revealed after thermal treatment of $58^{\circ} \mathrm{C}$ for $1.5 \mathrm{~min}$ [Fig. 3(b)]. Upon further thermal treatment from 1.5 to $7.5 \mathrm{~min}$ [Figs. 3(b)-3(f)], the bands of denatured collagen widen while the bands of unaltered collagen narrow. The denatured bands of

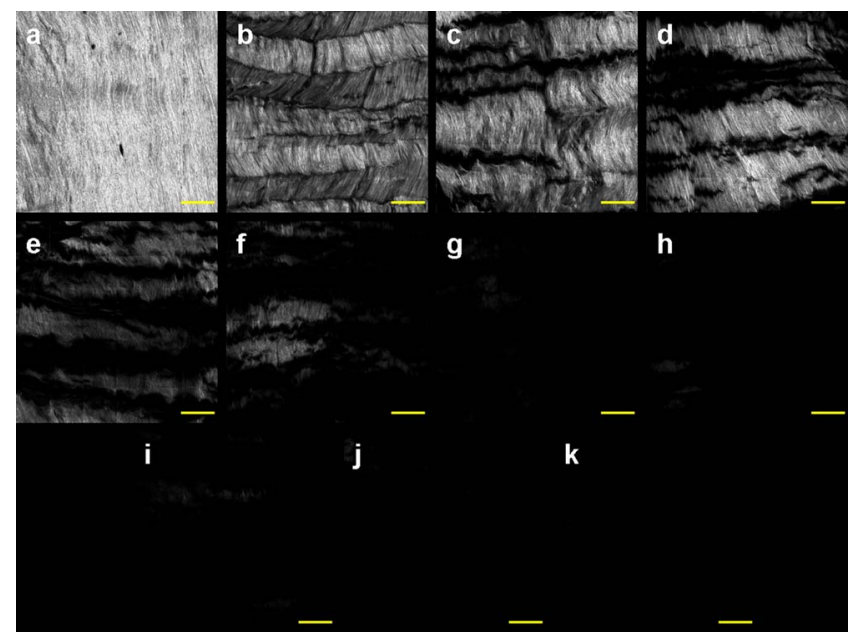

Fig. $3 \mathrm{SHG}$ images of rat tail tendons after thermal treatment at $58^{\circ} \mathrm{C}$ for (a) 0, (b) 1.5, (c) 3, (d) 4.5, (e) 6, (f) 7.5, (g) 9, (h) 10.5, (i) 12, (j) 13.5, and (k) 15 min (bars $100 \mu \mathrm{m})$. 


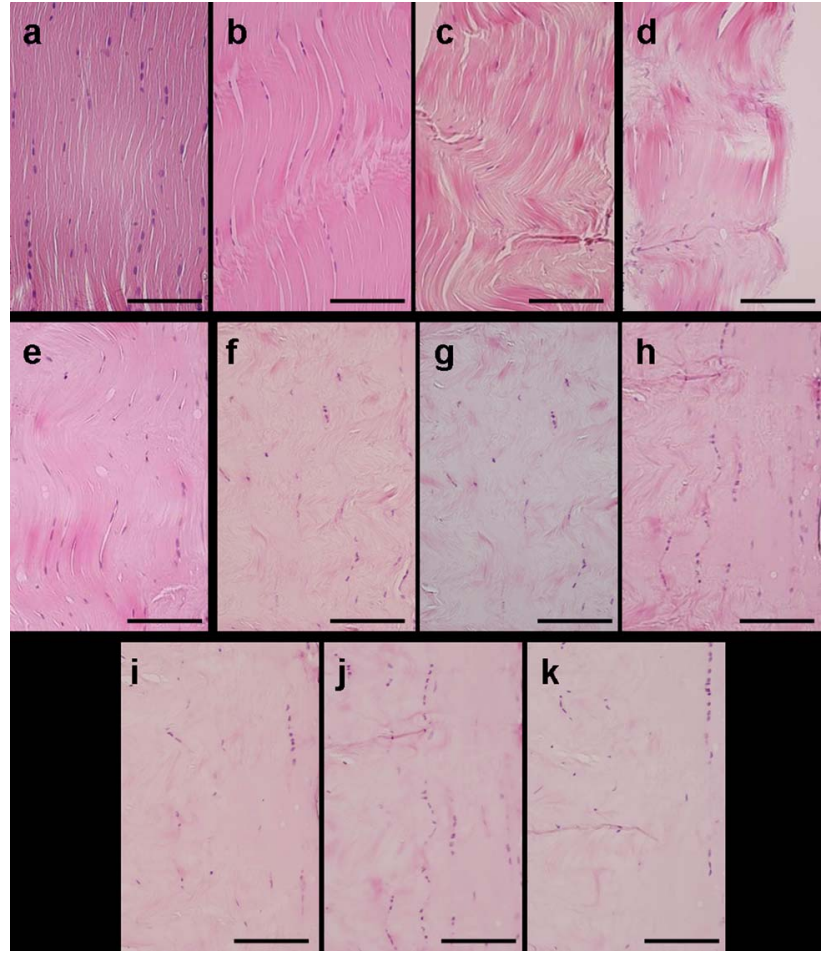

Fig. 4 Representative histological images of rat tail tendon after thermal treatment at $58{ }^{\circ} \mathrm{C}$ for (a) 0, (b) 1.5, (c) 3, (d) 4.5, (e) 6, (f) 7.5, (g) 9, (h) 10.5, (i) 12, (j) 13.5, and (k) 15 min (bars $100 \mu \mathrm{m}$ ).

collagen progressively replace the adjacent bands of unaltered collagen, and the bands of denatured collagen become confluent after thermal treatment for 9 min [Fig. 3(g)]. The SHG image remains mostly invisible upon further thermal treatment up to 15 min [Figs. 3(h)-3(k)]. Similarly, homogenized changes of collagen can be revealed in the histology of specimens heated for 9 to 15 min [Figs. 4(h)-4(k)]. A comparison of the SHG and histological images reveals the ability of SHG imaging to provide a sharper contrast between the unaltered and denatured collagen zones.

By comparing Figs. 2 and 3, the progressive decrease in SHG signals in Fig. 2 can be explained by the increased portion of denatured collagen in Fig. 3. It is important to appreciate the tiger-tail-like interrupted pattern of collagen denaturation observed in the SHG images. By judging the portion of denatured collagen in SHG images, we can obtain a rough idea of the proportion of denatured collagen during thermal treatment.

\subsection{Mathematical Model for Prediction of Collagen Shrinkage}

Since both the degree of collagen shrinkage and the decrease in SHG intensity show a dependence on duration of thermal treatment from 0 to $9 \mathrm{~min}$, there may be a correlation between collagen shrinkage and $\mathrm{SHG}$ intensities. Figure 5 shows the association of collagen shrinkage and SHG intensity during thermal treatment at $58^{\circ} \mathrm{C}$ for up to $15 \mathrm{~min}$, and the linear regression is calculated using linear least-square fits. As shown in Fig. 5, when mean rat tail tendon lengths during thermal treatment are plotted as a function of mean

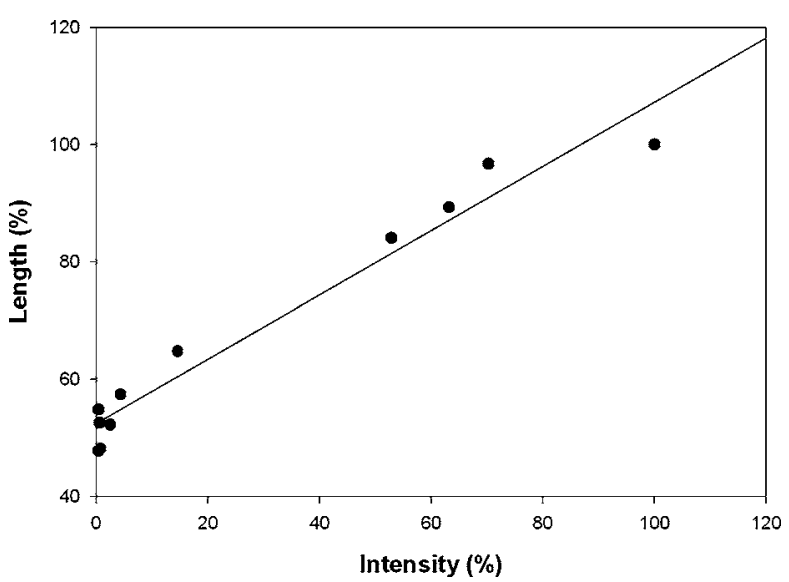

Fig. 5 Linear regression of mean collagen shrinkage and mean SHG intensity from thermal treatment of $58{ }^{\circ} \mathrm{C}$ for 0 to $15 \mathrm{~min}$. Mean rat tail tendon length (shown as percentage of initial length) is plotted as a function of mean SHG intensity (shown as percentage of initial intensity). The solid line is linear least-square fit.

SHG intensities, a good linear fit can be obtained $\left(r^{2}\right.$ $=0.9576$ ). The equation of the correlation between rat tail tendon length (percent of initial length) and SHG intensity (percent of initial intensity) calculated from the linear leastsquare fit is:

$$
Y=0.547 * X+52.5
$$

where $Y$ is the rat tail tendon length after thermal treatment (expressed as percent of initial length); and $X$ is the SHG intensity after thermal treatment (expressed as percent of percent of initial intensity).

Further, our data suggest that the denatured collagen has an ultimate length approximately $51 \%$ of the initial length (the average of tendon lengths at 9, 10.5, 12, 13.5, and $15 \mathrm{~min}$ ). This result is also consistent with a previous report showing that bovine joint capsule collagen has an ultimate length of about $50 \%$ of its initial length from thermal treatment. ${ }^{32}$ Assuming the linear dependence of collagen shrinkage on SHG intensity, depicted by Eq. (1), we can better quantify the correlation of collagen shrinkage with the changes in SHG intensity using the following equation:

$$
L_{d}=\left(0.547 I_{d} / I_{0}+0.525\right) * L_{0}
$$

where $L_{d}$ is the rat tail tendon length after thermal treatment; $L_{0}$ is the initial rat tail tendon length before thermal treatment; $I_{d}$ is the SHG intensity after thermal treatment; and $I_{0}$ is the initial SHG intensity before thermal treatment.

By the use of Eq. (2), there is a linear dependence of collagen shrinkage on the decrease of SHG intensity from thermal treatment before the collagen is fully denatured. When the collagen is fully denatured, as revealed by the absence of SHG signals, there is an ultimate length of collagen fibers of $52.5 \%$ of the initial length. Hence, by comparing the initial SHG intensity and final SHG intensity, we can easily predict the degree of collagen shrinkage during thermal treatment. 
Lin et al.: Prediction of heat-induced collagen shrinkage...

\section{Discussion}

In the traditional approach, collagen denaturation is investigated by use of histological examination, electron microscopy, and biochemical assays. ${ }^{12,17}$ In the case of biochemical assays, methodologies were developed to investigate the kinetic denaturation of collagen. Changes in viscosity can reflect the relative concentration of native and denatured states of collagen in the liquid states. ${ }^{33,34}$ However, these methods are not appropriate for real-time in-vivo monitoring, because they involve the removal of tissue specimens and additional processing before the extent of collagen denaturation can be determined. In this work, we have succeeded in monitoring the collagen denaturation process by use of SHG microscopy. Both morphological information and quantitative analysis can be obtained, and correlation with the gross tissue shrinkage can be made.

Heat-induced collagen denaturation depends both on the temperature used and the duration of thermal treatment. ${ }^{15} \mathrm{At}$ higher temperatures, the denaturation process progresses at faster rates. However, the mechanical load of the tissue and chemical modification of collagen also affect the denaturation process. ${ }^{12,15,35}$ Cross-links between collagen molecules contribute to the stability of collagen structures, ${ }^{36,37}$ and it has been reported that the cross-linking of collagen in tissue varies due to aging, diseased states, and environmental exposure. $^{38-41}$ Underheating may lead to compromised treatment responses, while overheating can lead to unfavorable results. In terms of thermal treatment in clinical applications, it is quite possible that the response of collagen to heating can be affected by the age, anatomical distribution, and other associated diseases in the patients. This may account for the different responses of patients to the same heating protocol. ${ }^{11}$ Therefore, the development of a real-time imaging modality to monitor the response to thermal treatment would contribute greatly to individualized treatment.

Although the detailed underlying mechanisms coupling collagen denaturation and tissue shrinkage are not fully characterized, it has been shown that gross shrinkage of collagen fibers provides much of the same information on the collagen denaturation kinetics. ${ }^{17}$ Therefore, our results suggest that the quantitative determination of the denatured collagen in the tissue from SHG imaging can be used to estimate the extent of collagen shrinkage and the degree of tissue damage.

The progressive loss of SHG in rat tails during thermal treatment indicates that the collagen structures responsible for SHG signals become gradually disrupted. Since there is a linear correlation between collagen shrinkage and the changes in overall SHG intensities, the approach proposed in this work enables us to predict collagen shrinkage by determining the changes in the sample's SHG intensities.

Our study also shows that after SHG signals completely fade, further heating is inefficient in shrinking the collagen. It seems that rat tail tendon reaches its ultimate shrinkage when its SHG signal completely fades. Further thermal treatment can at most shrink the collagen tissue by a small amount. This is consistent with a previous report that there is a terminal length of collagen shrinkage, even if the tissue is heated at different temperatures. ${ }^{15}$ Hence, thermal treatment beyond this point can theoretically further weaken the tensile strength of collagen tissue without achieving more tightening effects. $^{42-44}$

In addition to SHG intensity, SHG images also provide information on the collagen denaturation process. It is important to appreciate the use of large-area scanning in our study. The interrupted pattern of collagen denaturation in rat tail tendon cannot be easily identified when an image covering a small domain is used. In the case of rat tail tendon, a minimal scanning area of $500 \times 500 \mu \mathrm{m}^{2}$ is required to effectively monitor the denaturation process in detail. By examining the SHG images, we can visualize the extent of collagen denaturation and estimate the shrinkage. Compared with histological results, the SHG image provides sharper contrast in distinguishing the regions of preserved collagen from the denatured tissue.

\section{Conclusion}

In conclusion, shrinkage of thermally treated rat tail tendon at $58{ }^{\circ} \mathrm{C}$ shows a linear correlation with the decrease of SHG intensity. Our results show that SHG imaging is an effective method in predicting the shrinkage of thermally treated collagen and in monitoring the mechanisms of collagen denaturation. Our results suggest that quantitative SHG microscopy can be developed into a noninvasive method to monitor the thermal treatment of collagen in medical practices.

\section{Acknowledgment}

This work is supported by the National Research Program of Genomic Medicine of the National Science Council, Taiwan (NSC 94-3112-B-002-015-Y and NSC 93-3112-B-002-034).

\section{References}

1. T. R. Lyons, P. L. Griffith, F. H. Savoie, and L. D. Field, "Laserassisted capsulorrhaphy for multidirectional instability of the shoulder," Arthroscopy: J. Relat. Surg. 17, 25-30 (2001).

2. M. B. McDonald, P. S. Hersh, E. E. Manche, R. K. Maloney, J. Davidorf, M. Sabry, and the Conductive Keratoplasty United States Investigators Group, "Conductive keratoplasty for the correction of low to moderate hyperopia: U.S. flinical trial 1-year results on 355 eyes," Ophthalmology 109, 1978-1989 (2002).

3. R. E. Fitzpatrick, M. P. Goldman, N. M. Satur, and W. D. Tope, "Pulsed carbon dioxide laser resurfacing of photoaged facial skin," Arch. Dermatol. 132, 395-402 (1996).

4. T. S. Alster and S. Garg, "Treatment of facial rhytides with a highenergy pulsed carbon dioxide laser," Plast. Reconstr. Surg. 98, 791794 (1996).

5. K. A. Khatri, E. V. Ross, J. M. Grevelink, C. M. Magro, and R. R. Anderson, "Comparison of erbium: YAG and carbon dioxide lasers in resurfacing of facial rhytides," Arch. Dermatol. 135, 391-397 (1999).

6. D. J. Goldberg, "Full-face nonablative dermal remodeling with a 1320 nm Nd:YAG laser," Dermatol. Surg. 26, 915-918 (2000).

7. E. L. Tanzi, C. M. Williams, and T. S. Alster, "Treatment of facial rhytides with a nonablative $1450 \mathrm{~nm}$ diode laser: a controlled clinical and histologic study," Dermatol. Surg. 29, 124-128 (2003).

8. J. R. Lupton, C. M. Williams, and T. S. Alster, "Nonablative laser skin resurfacing using a $1540 \mathrm{~nm}$ erbium:glass laser: a clinical and histologic analysis," Dermatol. Surg. 28, 833-835 (2002).

9. D. J. Goldberg and K. B. Cutler, "Nonablative treatment of rhytides with intense pulsed light," Lasers Surg. Med. 26, 196-200 (2000).

10. T. S. Alster and E. Tanzi, "Improvement of neck and cheek laxity with a nonablative radiofrequency device: A lifting experience," Dermatol. Surg. 30, 503-507 (2004).

11. R. Fitzpatrick, R. Geronemus, D. Goldberg, M. Kaminer, S. Kilmer, and J. Ruiz-Esparza, "Multicenter study of noninvasive radiofrequency for periorbital tissue tightening," Lasers Surg. Med. 33, 232242 (2003)

12. N. T. Wright and J. D. Humphrey, "Denaturation of collagen via 
heating: An irreversible rate process," Аnnu. Rev. Biomed. Eng. 4 109-128 (2002).

13. J. Uitto, L. Pulkkinen, and M. L. Chu, "Collagen," in Fitzpatrick's Dermatology in General Medicine, 6th ed., I. M. Freedberg, A. Z. Eisen, K. Wolff, K. F. Austen, L. A. Goldsmith, and S. I. Katz, Eds., pp. 165-179, McGraw-Hill, New York (2003).

14. K. Pankhurst, "Incipient shrinkage of collagen and gelatin," Nature (London) 159, 538 (1947).

15. C. E. Weir, "Rate of shrinkage of tendon collagen- heat, entropy, and free energy of activation of the shrinkage of untreated tendon. Effect of acid, salt, pickle, and tannage on the activation of tendon collagen," J. Am. Leather. Chem. Assoc. 44, 108-140 (1949).

16. H. Hormann and H. Schlebusch, "Reversible and irreversible denaturation of collagen fibers," Biochemistry 10, 932-937 (1971).

17. K. Hayashi, G. Thabit III, J. J. Bogdanske, L. N. Mascio, and M. D. Markel, "The effect of nonablative laser energy on the ultrastructure of joint capsular collagen," Arthroscopy: J. Relat. Surg. 12, 474-481 (1996)

18. M. Rajadhyaksha, M. Grossman, D. Esterowitz, R. H. Webb, and R. R. Anderson, "In vivo confocal scanning laser microscopy of human skin: Melanin provides strong contrast," J. Invest. Dermatol. 104 946-952 (1995).

19. S. Hollingsworth, J. G. Bonshek, R. E., and N. Efron, "Correlation of the appearance of the keratoconic cornea in vivo by confocal microscopy and in vitro by light microscopy," Cornea 24, 397-405 (2005).

20. A. W. Drexler, U. Morgner, R. K. Ghanta, F. X. Kartner, J. S. Schuman, and J. G. Fujimoto, "Ultrahigh-resolution ophthalmic optical coherence tomography," Nat. Med. 7, 502-507 (2001).

21. A. Zoumi, A. Yeh, and B. J. Tromberg, "Imaging cells and extracellular matrix in vivo by using second-harmonic generation and twophoton excited fluorescence," Proc. Natl. Acad. Sci. U.S.A. 99, 11014-11019 (2002).

22. E. Brown, T. McKee, E. diTomaso, A. Pluen, B. Seed, Y. Boucher, and R. K. Jain, "Dynamic imaging of collagen and its modulation in tumors in vivo using second-harmonic generation," Nat. Med. 9, 796-800 (2003).

23. P. J. Campagnola and L. M. Loew, "Second-harmonic imaging microscopy for visualizing biomolecular arrays in cells, tissues and organisms," Nat. Biotechnol. 21, 1356-1360 (2003).

24. W. R. Zipfel, R. M. Williams, R. Christie, A. Y. Nikitin, B. T. Hyman, and W. W. Webb, "Live tissue intrinsic emission microscopy using multiphoton-excited native fluorescence and second harmonic generation," Proc. Natl. Acad. Sci. U.S.A. 100, 7075-7080 (2003).

25. K. König and I. Riemann, "High-resolution multiphoton tomography of human skin with subcellular spatial resolution and picosecond time resolution," J. Biomed. Opt. 8(3), 432-439 (2003).

26. P. Stoller, B. M. Kim, A. M. Rubenchik, K. M. Reiser, and L. B. Da Silva, "Polarization-dependent optical second-harmonic imaging of a rat-tail tendon," J. Biomed. Opt. 7(2), 205-214 (2002).

27. A. T. Yeh, B. Choi, J. S. Nelson, and B. J. Tromberg, "Reversible dissociation of collagen in tissues," J. Invest. Dermatol. 121, 13321335 (2003).

28. T. Yasui, Y. Tohno, and T. Araki, "Characterization of collagen orientation in human dermis by two-dimensional second-harmonicgeneration polarimetry," J. Biomed. Opt. 9(2), 259-264 (2004).

29. B. M. Kim, J. Eichler, K. M. Reiser, A. M. Rubenchik, and L. B. Da
Silva, "Collagen structure and nonlinear susceptibility: Effects of heat, glycation, and enzymatic cleavage on second harmonic signal intensity," Lasers Surg. Med. 27, 329-335 (2000).

30. S. J. Lin, R. J. Wu, H. Y. Tan, W. Lo, W. C. Lin, T. H. Young, C. J. Hsu, J. S. Chen, S. H. Jee, and C. Y. Dong, "Evaluating cutaneous photoaging by use of multiphoton fluorescence and second harmonic generation microscopy," Opt. Lett. 30, 2275-2277 (2005).

31. S. J. Lin, C. Y. Hsiao, Y. Sun, W. Lo, W. C. Lin, G. J. Jan, S. H. Jee, and C. Y. Dong, "Monitoring the thermally induced structural transitions of collagen using second harmonic generation microscopy," Opt. Lett. 30, 622-624 (2005).

32. G. S. Naseef III, T. E. Foster, K. Trauner, S. Solhpour, R. R. Anderson, and B. Zarins, "The thermal properties of bovine joint capsule: The basic science of laser- and radiofrequency-induced capsular shrinkage," Am. J. Sports Med. 25, 670-674 (1997).

33. H. Boedtker and P. Doty, "The native and denatured state of soluble collagen," J. Am. Chem. Soc. 78, 4267-4280 (1956).

34. R. E. Burge and R. D. Hynes, "The thermal denaturation of collagen in solution and its structural implications," J. Mol. Biol. 1, 155-164 (1959).

35. S. S. Chen, N. T. Wright, and J. D. Humphrey, "Heat-induced changes in the mechanics of a collagenous tissue: isothermal isotonic shrinkage," ASME J. Biomech. Eng. 120, 382-388 (1998).

36. R. B. Rucker and J. Murray, "Cross-linking amino acids in collagen and elastin," Am. J. Clin. Nutr. 31, 1221-1236 (1978).

37. C. A. Miles, N. C. Avery1, V. V. Rodin, and A. J. Bailey, "The increase in denaturation temperature following cross-linking of collagen is caused by dehydration of the fibers," J. Mol. Biol. 346, 551-556 (2005).

38. B. Buckingham and K. M. Reiser, "Relationship between the content of lysyl oxidase-dependent cross-links in skin collagen, nonenzymatic glycosylation, and long-term complications in type I diabetes mellitus," J. Clin. Invest. 86, 1046-1054 (1990).

39. M. Yamauchi, P. Prisayanh, Z. Haque, and D. T. Woodley, "Collagen cross-linking in sun-exposed and unexposed sites of aged human skin," J. Invest. Dermatol. 97, 938-941 (1991).

40. M. Tamura and O. Ishikawa, "An increase of mature type skin collagen cross-link, histidinohydroxylysinonorleucine, in the sclerotic skin of morphea," J. Dermatol. Sci. 25, 83-86 (2001).

41. J. Brinckmann, C. M. Neess, Y. Gaber, H. Sobhi, H. Notbohm, N Hunzelmann, P. P. Fietzek, P. K. Muller, J. Risteli, R. Gebker, and K. Scharffetter-Kochanek, "Different pattern of collagen cross-links in two sclerotic skin diseases: lipodermatosclerosis and circumscribed scleroderma," J. Invest. Dermatol. 117, 269-273 (2001).

42. K. Hayashi, G. Thabit III, A. C. Vailas, J. J. Bogdanske, A. J. Cooley, and M. D. Markel, "The effect of nonablative laser energy on joint capsular properties. An in vitro histologic and biochemical study using a rabbit model," Am. J. Sports Med. 24, 640-646 (1996).

43. K. Hayashi, G. Thabit III, K. L. Massa, J. J. Bogdanske, A. J. Cooley, J. F. Orwin, and M. D. Markel, "The effect of thermal heating on the length and histologic properties of the glenohumeral joint capsule," Am. J. Sports Med. 25, 107-112 (1997).

44. K. Hayashi and M. D. Markel, "Thermal modification of joint capsule and ligamentous tissues," Oper. Tech. Sports. Med. 6, 120-125 (1998). 